\title{
Impact of Confinement in Patients under Long-Term Noninvasive Ventilation during the First Wave of the SARS-CoV-2 Pandemic: A Remarkable Resilience
}

\author{
Chloé Cantero $^{a}$ Patrick Pasquina $^{a}$ Melissa Dominicé Dao $^{b}$ \\ Christine Cedraschi $^{c}$ Dan Adler ${ }^{a}$ Jérôme Plojoux ${ }^{a}$ Jean-Paul Janssens ${ }^{a}$ \\ ${ }^{a}$ Division of Pulmonary Diseases, Geneva University Hospitals, Geneva, Switzerland; ${ }^{\mathrm{b}}$ Division of Community \\ Medicine, Primary Care and Emergency Medicine, Geneva University Hospitals, Geneva, Switzerland; 'Division of \\ General Medical Rehabilitation and Multidisciplinary Pain Center, Division of Clinical Pharmacology and Toxicology, \\ Geneva University Hospitals, Geneva, Switzerland
}

\section{Keywords}

Noninvasive ventilation - COVID-19. SARS-CoV-2 .

Confinement - Mood disturbances - Depression · Anxiety ·

Chronic respiratory failure

\begin{abstract}
Background: During the first wave of the SARS-CoV-2 pandemic in Switzerland, confinement was imposed to limit transmission and protect vulnerable persons. These measures may have had a negative impact on perceived quality of care and symptoms in patients with chronic disorders. Objectives: To determine whether patients under long-term home noninvasive ventilation (LTHNIV) for chronic respiratory failure (CRF) were negatively affected by the 56-day confinement (March-April 2020). Methods: A questionnairebased survey exploring mood disturbances (HAD), symptom scores related to NIV (S ${ }^{3}$-NIV), and perception of health-care providers during confinement was sent to all patients under LTHNIV followed up by our center. Symptom scores and data obtained by ventilator software were compared between confinement and the 56 days prior to confinement. Results: Of a total of 100 eligible patients, 66 were included (median
\end{abstract}

karger@karger.com

(c) 2021 S. Karger AG, Basel

www.karger.com/res

Karger! age: 66 years [IQR: $53-74]): 35$ (53\%) with restrictive lung disorders, 20 (30\%) with OHS or SRBD, and 11 (17\%) with COPD or overlap syndrome. Prevalence of anxiety $(n=7 ; 11 \%)$ and depressive $(n=2 ; 3 \%)$ disorders was remarkably low. Symptom scores were slightly higher during confinement although this difference was not clinically relevant. Technical data regarding ventilation, including compliance, did not change. Patients complained of isolation and lack of social contact. They felt supported by their relatives and caregivers but complained of the lack of regular contact and information by health-care professionals. Conclusions: Patients under LTHNIV for CRF showed a remarkable resilience during the SARS-CoV-2 confinement period. Comments provided may be helpful for managing similar future health-care crises.

(c) 2021 S. Karger AG, Basel

\section{Introduction}

On February 25, 2020, the first case of SARS-CoV-2 infection was identified in Switzerland, 3 months after the beginning of the major pandemic, which has so far in- 
fected 61 million people worldwide and killed 1,439,784 persons (https://covid19.who.int/; November 28, 2020). Within the following weeks (March 14, 2020), the Swiss Federal Government imposed confinement measures for the entire population with a special focus on vulnerable subjects: this period lasted 56 days. The population was requested to stay at home; stores, restaurants, and public spaces were closed, and the local economy was almost put to a halt. For patients under home noninvasive ventilation (HMV) for chronic respiratory failure (CRF), confinement blocked access to elective procedures, such as routine follow-up visits dedicated to assessing efficacy of treatment and adequacy of settings, and compromised the feasibility of treatments provided at home such as rehabilitation or chest therapy. It also created anxiety not only among patients with chronic disorders but also among relatives, caregivers, and health-care workers [14] due to the high risk of infection but also because the activity of our public hospital was totally diverted to the care of COVID-19 patients.

Recent publications have shown, in other diseases and settings, how social isolation related to confinement during the COVID-19 pandemic has affected both patients and caregivers in many fundamental aspects of their everyday life. Impact on mental well-being, psychological strain/stress, anxiety, depression, increase in body weight, and changes in eating habits [5] have been reported in the general population [6-9] and in the elderly [10]. Both positive and negative impacts ("comfort food") on eating habits in the general population have been described [5, $6,11]$. Physical activity decreased in the general population, and conversely increased physical activity seemed to improve resilience $[12,13]$. Socioeconomic impact on the general population was also a major issue. In specific chronic disorders such as epilepsy $[14,15]$, various neuromuscular diseases [16], amyotrophic lateral sclerosis [1], Parkinson's disease [17], and spondyloarthritis [18], a negative impact of lockdown on symptom and/or disease control has been reported. Very little information however has been published to date concerning patients with chronic respiratory diseases.

The present study explores how patients under HMV for CRF perceived the confinement and its impact on their access to care. Our hypotheses were (1) that anxiety and depression would increase in this population during the confinement; (2) that use of noninvasive ventilation (NIV) could be affected in several ways: for instance, decreased compliance and tolerance to treatment, increase in symptoms and side effects related to NIV, and changes in pattern of breathing; (3) that delays may have occurred in solving NIV-related problems, and (4) that patients would express feelings such as fear, frustration, or even anger toward the medical system and complain of isolation.

\section{Materials and Methods}

In the Geneva area, patients under long-term HMV are cared for both by pulmonologists in private practice and by local hospitals, with the support of health care providers [19]. The present study focuses on patients under HMV who were followed up by the Division of Pulmonary Diseases of Geneva University Hospitals at the time of confinement. These patients usually have elective follow-up visits at our hospital (every 3-6 months) and home visits by specialized nurses either electively or on demand. Most patients are also telemonitored, and hotlines (weekdays and work hours) are available for HMV-related problems (technical support and specialized nurse).

At the beginning of the confinement, scheduled elective inhospital visits and home visits were canceled; however, hotlines were maintained. When appropriate (average daily use $>16 \mathrm{~h} /$ day), a second home ventilator was made available. Furthermore, all ventilator-dependent patients received spare tubings and interfaces to minimize the risk of technical problems.

Just after the confinement period, we designed a questionnairebased study to analyze the impact of confinement on this population. We explored 3 domains:

1. Symptoms and side effects related to NIV, using the $S^{3}$-NIV score, designed by our group [20] and used routinely during hospital visits in our center. This 11-item scale includes 2 subscores: "respiratory symptoms" and "sleep and NIV-related side effects" such as discomfort caused by leaks, interface, or xerostomia. The score ranges from 0 to 10 , and higher values indicate less symptoms or side effects related to NIV. When available, $\mathrm{S}^{3}$-NIV scores obtained prior to the confinement (within 6 months) were compared to values related to the confinement period.

2. Anxiety and depression were assessed using the Hospital Anxiety and Depression Scale (HADS) [21, 22]. This 14-item questionnaire includes 2 subgroups: 7 focused on anxiety and 7 on depression. The score ranges from 0 to 42 , and a value $<8$ is considered normal, between 8 and 10 is considered borderline, and $>10$ is suggestive of an anxiety and/or a depression disorder. Although not specifically designed for patients under HMV, this score has been widely used for documenting the prevalence of anxiety and depression disorders in patients with chronic respiratory disorders including patients with HMV and recently used as an anchor for quantifying the minimal clinically important difference of the Severe Respiratory Questionnaire [23-28].

3. A targeted questionnaire was devised with experts in qualitative assessment of care (coauthors Christine Cedraschi and Melissa Dominicé Dao) and tested on a pilot group of patients $(n=6)$ for coherence, feasibility, and relevance. It included the following:

- Twenty-four questions focusing on practical aspects related to home environment, home care, occurrence of infection (whether positive for COVID-19 or not), and technical problems related to NIV. 
- Thirteen Likert-type questions exploring items such as fear of COVID-19, impact of confinement on social relationships, isolation, and quality of sleep.

- Three open questions regarding their difficulties, expectations, and resources during the COVID-19 period.

As such, this led to a questionnaire with a total of 65 items. Data from ventilator software (ResScan ${ }^{\circledR}$ vers 6.0, ResMed; EncoreBasic $^{\circledR}$ vers 2.12, Philips Respironics; and DirectView ${ }^{\circledR}$ vers 2.4, Philips Respironics) covering the 56 days of confinement (i.e., leaks, tidal volume, apnea-hypopnea index [AHI], compliance, and daily use) were retrieved either through our telemonitoring platform or during home visits and compared to the 56 days preceding the confinement period. Questionnaires were sent by post to the patients' homes with a reminder after 1 month and then after 6 weeks. The study protocol was accepted by our local ethics committee (2020-01509) and registered at clinicaltrials.gov (NCT04477473).

\section{Statistics}

All data were described as frequency and percentage for qualitative data and as mean and standard deviation or median and interquartile range (IQR) for quantitative data. HADS scores measured just after the confinement were compared between diagnostic groups ([1] COPD and overlap syndrome; [2] restrictive lung disorders including neuromuscular disorders [NMD], and [3] obesity hypoventilation syndrome [OHS] and sleep-related breathing disorders [SRBD]) using a linear regression model. Data concerning $\mathrm{S}^{3}$-NIV were compared between both periods (before and during the confinement) using paired $t$ tests. Data provided by ventilator software were compared between periods using Wilcoxon signed rank tests with continuity corrections. Missing data were not replaced but simply reported to reflect the real-life nature of the study. Statistical significance was set at a 2-sided level of 5\% for all analyses. All analyses were performed using R software version R-4.0.2 (R Foundation for Statistical Computing, Vienna, Austria. URL https://www.R-project.org).

\section{Results}

The flowchart (Fig. 1) shows the recruitment process. The day before confinement, 117 patients under HMV were followed up by the Division of Pulmonary Diseases of Geneva University Hospitals. Four patients died during the confinement period, 3 of COVID-19 infection and 1 of non-COVID-19 infection, and thus could not be included. After exclusion of 13 other patients because of language or cognitive barriers, 100 patients were eligible and solicited for the present study. Thirty-four patients refused to participate or did not reply and 66 accepted and provided answers to the 3 questionnaires. Sixty-two patients of the 100 included were telemonitored (AirView ${ }^{\circledR}$; ResMed). All patients included were on NIV.

There was no significant difference between the 34 patients who refused inclusion and the study population in terms of age (included median: 59 [IQR: 46; 69] vs. 60 [48;

SARS-CoV-2 Pandemic Confinement in Patients on Home Mechanical Ventilation

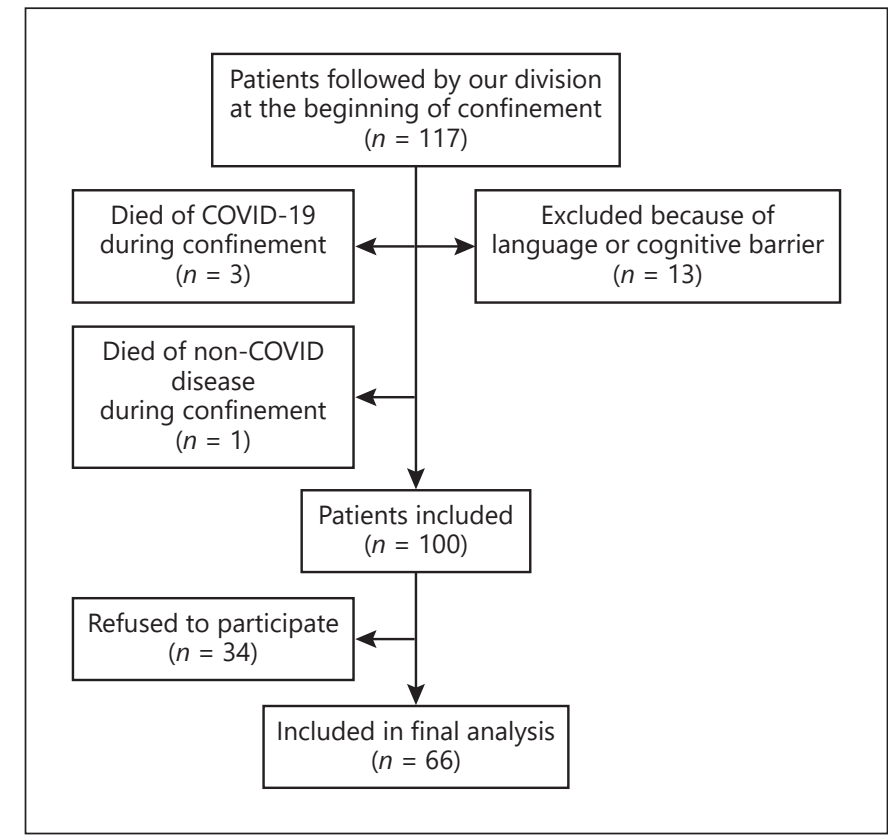

Fig. 1. Flowchart.

70]; $p=0.861$ ), gender (included $56 \%$ male vs. $62 \%$; $p=$ 0.739 ), and time spent in years under NIV (included median: 3.5 [IQR: $2.2 ; 8.3$ ] vs. 5.4 [IQR: 1.6 ; 9.9]; $p=0.845$ ). Distribution of diagnoses differed however with a participation rate of roughly $80 \%$ in patients with "COPDoverlap syndrome" and "restrictive lung disorders" (including NMD) versus only $50 \%$ in the "OHS and SRBD" group $(p=0.012)$.

Baseline characteristics of the study population are described in Table 1. NMD were the largest group in this study (details of diagnoses are given in online suppl. Table 1S; see www.karger.com/doi/10.1159/000516327 for all online suppl. material).

\section{$S^{3}$-NIV Score}

$S^{3}$-NIV scores were available in 42 patients before and during confinement (Table 2). Total score was slightly higher before confinement than during confinement (mean difference $=0.36$ [95\% CI: 0.04-0.69], $p=0.029$ ) while subscores did not differ significantly between periods. Missing values prior to confinement were mostly related to severely limited patients who had NMD or severe COPD: in these patients, elective follow-up visits were performed mostly at home, and $S^{3}$-NIV was not collected.

For all patients, $\mathrm{S}^{3}$-NIV scores provided just after the confinement $(n=66)$ were 7.1 (IQR: $5.4 ; 7.9)$ for "respira- 
Table 1. Baseline characteristics of study population at the first day of confinement $(n=66)$

\begin{tabular}{lc}
\hline Age, years & $66(53 ; 74)$ \\
Gender (male/female) & $29(44 \%) / 37(66 \%)$ \\
Diagnostic groups & \\
$\quad$ COPD and overlap syndrome & $11(17 \%)$ \\
Restrictive lung disorders & $35(53 \%)$ \\
$\quad$ NMD & $32(48 \%)$ \\
$\quad$ Other restrictive disorders* & $3(5 \%)$ \\
OHS and SRBD & $20(30 \%)$ \\
$\quad$ OHS & $9(14 \%)$ \\
$\quad$ SRBD & $10(17 \%)$ \\
Time under NIV, months & $69(33 ; 94)$ \\
Environment/autonomy & $11(17 \%)$ \\
Regular professional activity & $60(91 \%)$ \\
Can leave his/her home & $14(21 \%)$ \\
Lives alone & $3(4 \%)$ \\
Lives in a nursing home &
\end{tabular}

Values are expressed as median (IQR) or frequency (\%). COPD, chronic obstructive pulmonary disease; NMD, neuromuscular disorder; NIV, noninvasive ventilation; OHS, obesity hypoventilation syndrome; SRBD, sleep-related breathing disorders. * Posttuberculosis or kyphoscoliosis. ** Including with a wheelchair and with help.

tory symptoms" and 7.5 (IQR: 6; 9) for "sleep and side effects." The median total score was 7.5 (IQR: 5.9; 8).

\section{Hospital Anxiety and Depression Scale (HADS)}

Three patients did not answer all of the 14 questions. Among the remaining patients, 7 (11\%) had scores suggestive of an anxiety disorder (HAD-A >10) and $2(3 \%)$ of depression (HAD-D >10). Anxiety and depression scores did not differ significantly between diagnostic groups (online suppl. Fig. 1S). HAD scores were provided only for the confinement period.

\section{Technical Data Related to NIV}

Fifty-eight patients (88\%) used ResMed devices (S9 VPAP ST ${ }^{\circledR}$, Lumis $150^{\circledR}$, Stellar $150^{\circledR}$, or Astral $\left.150^{\circledR}\right)$, 5 (8\%) used Philips Respironics devices (BiPAP A $40^{\circledR}$ ), and 1 patient (1\%) an Air Liquide device (Monnal T50 ${ }^{\circledR}$ ). Data were missing for 2 patients.

During the confinement, most patients $(50 / 64,78 \%)$ used their ventilator every day, and only 4 patients $(6 \%)$ used their ventilator $<28$ days (i.e., $50 \%$ of the confinement period). This was similar to what was observed during the 56 days before confinement: $46 / 63$ patients (73\%) used their ventilator every day and only 2 patients $(3 \%)$ used their ventilator $<28$ days.
When patients used their ventilator, they used it on average 8.4 (SD 3.5) hours per day before and 8.6 (3.8) hours per day during the confinement (mean difference $=15$ min, 95\% CI: $0-30, p=0.050)$. Because data summaries (leaks, respiratory rate, and percentage of triggered breaths) provided by ventilator software differ between manufacturers, comparisons were made only for data from ResMed devices ( $n=58$; Table 3$)$. No significant difference was found for unintentional leaks, percentage of triggered breaths, backup respiratory rate, and apnea-hypopnea index (AHI) between both study periods (Table 3 ).

\section{Targeted Questionnaire}

None of the study patients was treated at home or hospitalized for a COVID-19 infection during the confinement, and 3 patients were hospitalized for a non-COVID-19 pulmonary infection. Four patients were treated at home for a lower respiratory tract infection according to a predefined action plan. None of the patients required an emergency home medical visit. No technical problem occurred with NIV devices and/or oxygen extractors. Few patients $(n=3 ; 5 \%)$ expressed concern about the possibility of a lack of technical support in case of problems with their NIV and/or oxygen extractor. During the confinement, elective evaluations of NIV were canceled in 24 cases (36\%), home visits by nurses in 19 cases (29\%), and home chest therapy or rehabilitation in 17 patients (26\%).

Approximately half of the patients surveyed $(n=36)$ provided free-text comments to at least one of the 3 open questions regarding their difficulties, expectations, and resources during the COVID-19 period (Table 4). The main difficulties expressed by our patients during the confinement period were having to stay homebound and lack of physical activities ( $n=9,28 \%$ of responses), lack of social contact with friends and family $(n=5,16 \%)$, and cancellation of their health-care appointments $(n=3$, 9\%). Patients related that outside help, contact with their loved ones ( $n=11,33 \%)$, keeping a normal daily rhythm and regular physical activity $(n=5,15 \%)$, and finding a source of distraction $(n=4,12 \%)$ were all helpful to them to maintain good physical and mental health. Patients spontaneously made grateful and positive comments on the care and information provided by their health professionals during confinement $(n=14,58 \%)$ although a few wished for more phone contact and information regarding contagion ( $n=6,25 \%)$. A selection of relevant freetext comments is provided in Table 4 . 
Table 2. $S^{3}$-NIV scores during the 56 days prior to the confinement period and during the confinement

\begin{tabular}{|c|c|c|c|c|}
\hline \multicolumn{5}{|l|}{ S3-NIV scores $(n=42)$} \\
\hline Respiratory symptoms score & $7.2(1.5)$ & $6.8(1.5)$ & $0.41(-0.06$ to 0.87$)$ & 0.084 \\
\hline NIV side effects score & $7.7(1.6)$ & $7.4(1.9)$ & $0.31(-0.11$ to 0.73$)$ & 0.148 \\
\hline
\end{tabular}

Table 3. Data provided by ventilator software during the 56 days prior to the confinement period and during the confinement

\begin{tabular}{|c|c|c|c|c|c|}
\hline \multicolumn{6}{|c|}{ Data provided by ventilator software (ResMed devices, $n=58)$} \\
\hline Unintentional leaks, L/min & $3.5(0.1 ; 9.6)$ & 5 & $5.0(0.1 ; 8.9)$ & 4 & 0.218 \\
\hline Backup respiratory rate, cycles/min & $18(15 ; 18.5)$ & 3 & $17(14.8 ; 19)$ & 2 & 0.742 \\
\hline Spontaneous inspirations, $\%$ & $23(12 ; 54)$ & 9 & $24(10 ; 57)$ & 8 & 0.585 \\
\hline
\end{tabular}

Values are expressed as median (IQR) or frequency (\%); data were available for both periods in 58 patients using ResMed devices. AHI, apnea-hypopnea index; $n$, number of patients. * Wilcoxon signed rank test with continuity correction.

\section{Discussion}

This study explored the perception of confinement related to the COVID-19 pandemic and its impact on health care in patients under long-term home NIV. The response rate to our mailing was high (two-thirds of patients solicited) for this type of study. Among respondents to the questionnaire, we noticed that the prevalence of anxiety (11\%) and depression (3\%) was remarkably low. When compared to baseline values, the confinement period was associated with minor increases in symptoms of CRF or reported side effects of NIV. Respiratory rate, unintentional leaks, apnea-hypopnea index, and compliance to NIV, all markers of appropriate use of the ventilator and efficacy downloaded from the ventilator, were unchanged. Daily use of the ventilator increased on average by $15 \mathrm{~min}$ during the confinement, which was not considered as clinically relevant. Targeted questions revealed that home treatments (nurse visits, chest therapy, and rehabilitation) were interrupted in a quarter of respondents, and elective evaluations were canceled or postponed in close to $40 \%$. Technical problems were rare. Patients mentioned the weight of social isolation and the importance of their close relatives as a psychological support.

SARS-CoV-2 Pandemic Confinement in Patients on Home Mechanical Ventilation
We had anticipated a higher prevalence of anxiety and depression and a more important impact of social isolation on compliance to NIV and symptom scores. This was not the case. Indeed, the impact of isolation and decreases in social interactions and relationships on morbidity and mortality has been documented in subjects with preexisting health conditions [29-31]. Although patients did mention social isolation as a problem, HAD scores were well within or below published values for patients under HMV, with a remarkably low prevalence of anxiety or depressive disorders in spite of the pandemic [27, 32-37]. Interestingly, a Spanish study which included 100 patients with COPD reported that patients' feelings regarding their lung disease and general health were similar or even better during lockdown [38]. There are a few plausible contributive factors. Patients on HMV are most often sedentary and very few have a regular (often part-time) professional activity ( $17 \%$ in our study population). Also, although $90 \%$ of patients can leave their home, it is for many neuromuscular patients ( $48 \%$ of the study population), a complicated and sometimes tedious exercise. Furthermore, even if some therapeutic interventions were discontinued during the confinement, many neuromuscu- 
Table 4. Summary of response categories provided to open questions regarding coping with the confinement and perception of health care

Thematic categories of responses

Illustrative examples

During this COVID-19 period, what difficulties did you encounter to maintain your mental and physical health care? ( $n=32$ comments)

Being homebound $(n=9)$

Always being at home and not able to do my usual activities

Not being able to go out, I was locked up $24 \mathrm{~h}$ a day

Feeling isolated (from the outside world, family, and friends, The lack of contact with the outside world

$n=5)$

To be isolated from my family and not having seen my granddaughter since her birth

Interference with regular treatments: postponing elective tests, interruption of rehabilitation, lack of medication, limiting caregivers at home $(n=3)$

The halt of my physiotherapy program

Not having enough drugs for my back pain because I was too afraid to go outside

None $(n=10)$

Nothing special

It didn't change much my daily life

During this COVID-19 period, what were your expectations from your health-care professionals? $(n=24$ comments)

Desire for more information about the risks related to SARS- To call to see if everything was going well

$\mathrm{CoV}-2$ and requests for regular contact/phone calls $(n=6)$

To be informed if I was to be considered as a person at risk for coronavirus and what could be the consequences of a contamination

Spontaneous expressions of positive comments and gratitude May God protect all doctors and nurses toward health-care professionals during the pandemic $(n=$ 14)

I congratulate all the health professionals who risked their life for others. Thank you, you are heroes!

During this COVID-19 period, what helped you maintain your mental and physical health? $(n=33$ comments $)$

Maintaining a normal rhythm and regular physical activity $(n=5)$

Having a rhythm

To walk an hour in the morning

Support from family and close relatives $(n=11)$

My relatives took care of me and my shopping

The support of my spouse and family

Staying busy and entertained $(n=2)$

Entertainment such as television and computer

Read and philosophize

Support received by health-care professional caregivers $(n=$ My doctors were (virtually) very present to advise and monitor me and asking regularly how I was doing

Dr. X answered my mom's email

Adherence to expert health-care recommendations $(n=5) \quad$ Staying at home

I just respected the basic hygiene rules at home and at work

Spirometry. Various aerosols

Finding safe solutions to maintain physical activity $(n=2)$

Walk early in the morning in front of my house for $30 \mathrm{~min}$

lar patients rely on a group of caregivers which in most cases did not change. Therefore, the real impact on the environment of neuromuscular patients may have been limited.
Symptom scores were also within expected values for this population [20]. There was a minor statistically significant decrease in the total $S^{3}$-NIV score during the confinement. Subscores of the $S^{3}$-NIV did not show, how- 
ever, any significant increase in perceived side effects of NIV or respiratory symptoms.

The open questions provided important additional insights. Patients found difficult the confinement per se, the isolation, and the resulting lack of contact with the outside world. Lack of exercise, physiotherapy, and rehabilitation was also problematic. Although there were many positive comments regarding health-care professionals, several patients would have liked more information and contact: they expressed their desire for regular phone calls by HCW, for being updated on the COVID-19 situation and how it impacted on their situation, for reassurance as to the persisting availability of technical support and equipment. Our focus at the beginning of the first wave of the pandemic was mostly on logistics: providing enough backup equipment (extra interfaces, tubings, filters, and low threshold for a backup ventilator) and information (hotlines) in case of technical problems. This suggests that some form of teleconsultation should be added to the well-accepted telemonitoring, implemented routinely in most patients. The support provided by close relatives, family members, and caregivers and HCW was also of major importance.

There are a few limitations to this pragmatic observational study: first, the distribution of diagnoses in the study population differed from larger studies of NIV in our area [19]: almost half of them had NMD, and all patients had been on HMV for a prolonged period. The lower participation rate of patients with OHS and SRBD may have affected results concerning SARS-CoV-2 morbidity and mortality since OHS patients are a high-risk group for COVID-19 infection. This case selection is related to our focus, as a University Hospital, on more complex cases, with a higher burden placed on caregivers. This may have had an impact on our results.

Second, we could not compare $S^{3}$-NIV scores before and during confinement for 24 patients: $\mathrm{S}^{3}$-NIV is not always collected during home visits (more frequent in NMD patients). $S^{3}$-NIV scores obtained for all patients during the confinement are quite close to those collected prior to confinement, suggesting that significant changes were unlikely.

Third, these results are related to specific standards of care in our area and to geographic and socioeconomic characteristics of Geneva. Extrapolating these results to other environments and ethnic/socioeconomic groups may not be possible. This is also the case for patients ventilated by tracheostomy, none of whom were included in this study: home invasive ventilation is a very rare occurrence in our area [19].

SARS-CoV-2 Pandemic Confinement in Patients on Home Mechanical Ventilation
Fourth, we chose to use the HADS as a questionnaire designed for screening for anxiety and depression in chronic disorders. Although not specifically designed for HMV patients, it is widely used in these patients by expert groups, simple to perform, and short [24-27, 32, 35, 36]. We did not add a questionnaire such as the Severe Respiratory Insufficiency (SRI) questionnaire [39], designed for assessing health-related quality of life in patients under HMV. This was a strategic choice: we feared that adding 49 questions to the 65 items already submitted to our patients might compromise the response rate and/or the quality of the responses. Also, HRQL per se was not the main focus of this study, even if several items contributing to HRQL were assessed through the use of the HADS and the $S^{3}$-NIV. The SRI could have contributed additional information on HRQL, through a more detailed assessment for instance of activities of daily life, psychosocial aspects, and symptoms. Finally, this study focused on the first wave of the pandemic in our area: the second wave which has hit most countries worldwide may prove to be more difficult to accept because of the fatigue, the lassitude by this persistent situation, and the impact on families, caregivers, and health-care workers.

\section{Conclusion}

The combination of a low prevalence of anxiety and depression, favorable symptom scores, and excellent and stable compliance suggests a remarkable resilience during the first wave of the SARS-CoV-2 pandemic in this vulnerable population of patients under HMV for CRF. Suggestions provided by the patients such as providing more regular phone contacts and information regarding SARS$\mathrm{CoV}-2$ in their particular situation may be helpful during the second wave of the pandemic.

\section{Acknowledgement}

We are grateful to Antoine Poncet for the statistical analyses and his thorough revision of the manuscript.

\section{Statement of Ethics}

The studies involving human participants were reviewed and approved by the Cantonal Commission for Research Ethics (CCER) in Geneva, Switzerland (2020-01509), in agreement with the amended Declaration of Helsinki. The patients/participants provided their written informed consent to participate in this study. 


\section{Conflict of Interest Statement}

The authors declare that they have no conflicts of interest.

\section{Funding Sources}

The authors declare that no funding was provided for this study.

\section{Author Contributions}

Chloé Cantero, Patrick Pasquina, and Jean-Paul Janssens contributed to the conception and design of the study. Chloé Cantero and Patrick Pasquina organized the database. Chloé Cantero and Jean-Paul Janssens wrote the first draft of the manuscript. Chloé Cantero, Patrick Pasquina, Melissa Dominicé Dao, Christine Cedraschi, Dan Adler, Jérôme Plojoux, and Jean-Paul Janssens wrote sections of the manuscript. All authors contributed to manuscript revision and read and approved the submitted version.

\section{References}

1 De Lucia N, Ausiello FP, Spisto M, Manganelli F, Salvatore E, Dubbioso R. The emotional impact of COVID-19 outbreak in amyotrophic lateral sclerosis patients: evaluation of depression, anxiety and interoceptive awareness. Neurol Sci. 2020;41(9):2339-41.

2 Alexopoulos P, Soldatos R, Kontogianni E, Frouda M, Loanna Aligianni S, Skondra M, et al. COVID-19 crisis effects on caregiver distress in neurocognitive disorder. J Alzheimers Dis. 2020;79(1):459-66.

3 Cohen G, Russo MJ, Campos JA, Allegri RF. Living with dementia: increased level of caregiver stress in times of COVID-19. Int Psychogeriatr. 2020;32:1377-81.

4 Lefevre H, Stheneur C, Cardin C, Fourcade L, Fourmaux C, Tordjman E, et al. The bulle: support and prevention of psychological decompensation of health care workers during the trauma of the COVID-19 epidemic. J Pain Symptom Manage. 2021;61(2):416-22.

5 Di Renzo L, Gualtieri P, Cinelli G, Bigioni G, Soldati L, Attina A, et al. Psychological aspects and eating habits during COVID-19 home confinement: results of EHLC-COVID-19 Italian online survey. Nutrients. 2020;12(7): 2152.

6 Ammar A, Brach M, Trabelsi K, Chtourou H, Boukhris O, Masmoudi L, et al. Effects of COVID-19 home confinement on eating behaviour and physical activity: results of the ECLB-COVID19 international online survey. Nutrients. 2020;12(6):1583.

7 Ammar A, Mueller P, Trabelsi K, Chtourou $\mathrm{H}$, Boukhris O, Masmoudi L, et al. Psychological consequences of COVID-19 home confinement: the ECLB-COVID19 multicenter study. PLoS One. 2020;15(11): e0240204.

8 Odriozola-Gonzalez P, Planchuelo-Gomez A, Irurtia MJ, de Luis-Garcia R. Psychological symptoms of the outbreak of the COVID-19 confinement in Spain. J Health Psychol. 2020. Epub ahead of print.

9 Planchuelo-Gomez A, Odriozola-Gonzalez P, Irurtia MJ, de Luis-Garcia R. Longitudinal evaluation of the psychological impact of the COVID-19 crisis in Spain. J Affect Disord. 2020;277:842-9.
10 Sepulveda-Loyola W, Rodriguez-Sanchez I, Perez-Rodriguez P, Ganz F, Torralba R, Oliveira DV, et al. Impact of social isolation due to COVID-19 on health in older people: mental and physical effects and recommendations. J Nutr Health Aging. 2020;24(9):93847.

11 Rodriguez-Perez C, Molina-Montes E, Verardo V, Artacho R, Garcia-Villanova B, GuerraHernandez EJ, et al. Changes in dietary behaviours during the COVID-19 outbreak confinement in the Spanish COVIDiet study. Nutrients. 2020;12(6):1730.

12 Carriedo A, Cecchini JA, Fernandez-Rio J, Mendez-Gimenez A. Resilience and physical activity in people under home isolation due to COVID-19: a preliminary evaluation. Ment Health Phys Act. 2020;19:100361.

13 Carriedo A, Cecchini JA, Fernandez-Rio J, Mendez-Gimenez A. COVID-19, psychological well-being and physical activity levels in older adults during the nationwide lockdown in Spain. Am J Geriatr Psychiatry. 2020; 28(11):1146-55.

14 Fonseca E, Quintana M, Lallana S, Luis Restrepo J, Abraira L, Santamarina E, et al. Epilepsy in time of COVID-19: a survey-based study. Acta Neurol Scand. 2020;142(6):545-54.

15 Sanchez-Larsen A, Gonzalez-Villar E, DiazMaroto I, Layos-Romero A, Martinez-Martin $\mathrm{A}$, Alcahut-Rodriguez $\mathrm{C}$, et al. Influence of the COVID-19 outbreak in people with epilepsy: analysis of a Spanish population (EPICOVID registry). Epilepsy Behav. 2020;112:107396.

16 Mauri E, Abati E, Musumeci O, Rodolico C, D'Angelo MG, Mirabella M, et al. Estimating the impact of COVID-19 pandemic on services provided by Italian Neuromuscular Centers: an Italian Association of Myology survey of the acute phase. Acta Myol. 2020; 39(2):57-66.

17 Kumar N, Gupta R, Kumar H, Mehta S, Rajan $\mathrm{R}$, Kumar D, et al. Impact of home confinement during COVID-19 pandemic on sleep parameters in Parkinson's disease. Sleep Med. 2020;77:15-22.

18 Roux CH, Brocq O, Gerald F, Pradier C, Bailly L. Impact of home confinement during the COVID-19 pandemic on medication use and disease activity in spondyloarthritis patients. Arthritis Rheumatol. 2020;72(10):1771-2.
19 Cantero C, Adler D, Pasquina P, Uldry C, Egger B, Prella $\mathrm{M}$, et al. Long term noninvasive ventilation in the Geneva Lake area: indications, prevalence and modalities. Chest. Forthcoming 2020

20 Dupuis-Lozeron E, Gex G, Pasquina P, Bridevaux PO, Borel JC, Soccal PM, et al. Development and validation of a simple tool for the assessment of home noninvasive ventilation: the S(3)-NIV questionnaire. Eur Respir J. 2018;52(5):1801182.

21 Lepine JP, Godchau M, Brun P, Lemperiere T. [Evaluation of anxiety and depression among patients hospitalized on an internal medicine service]. Ann Med Psychol (Paris). 1985; 143(2):175-89.

22 Zigmond AS, Snaith RP. The hospital anxiety and depression scale. Acta Psychiatr Scand. 1983;67(6):361-70

23 Raveling T, Kort J, Bladder G, Windisch W, Wijkstra PJ, Duiverman ML. The minimal clinically important difference of the Severe Respiratory Insufficiency questionnaire in severe COPD. Eur Respir J. 2020;56(6):2001334.

24 Duiverman ML, Vonk JM, Bladder G, van Melle JP, Nieuwenhuis J, Hazenberg A, et al. Home initiation of chronic non-invasive ventilation in COPD patients with chronic hypercapnic respiratory failure: a randomised controlled trial. Thorax. 2019;75(3):244-52.

25 Duiverman ML, Wempe JB, Bladder G, Jansen DF, Kerstjens HA, Zijlstra JG, et al. Nocturnal non-invasive ventilation in addition to rehabilitation in hypercapnic patients with COPD. Thorax. 2008;63(12):1052-7.

26 Duiverman ML, Wempe JB, Bladder G, Kerstjens HA, Wijkstra PJ. Health-related quality of life in COPD patients with chronic respiratory failure. Eur Respir J. 2008;32(2): 379-86.

27 Crescimanno G, Greco F, D’Alia R, Messina L, Marrone O. Quality of life in long term ventilated adult patients with Duchenne muscular dystrophy. Neuromuscul Disord. 2019; 29(8):569-75.

28 Janssens JP, Penalosa B, Degive C, Rabeus M, Rochat T. Quality of life of patients under home mechanical ventilation for restrictive lung diseases: a comparative evaluation with COPD patients. Monaldi Arch Chest Dis. 1996;51(3):178-84. 
29 Holt-Lunstad J. Why social relationships are important for physical health: a systems approach to understanding and modifying risk and protection. Annu Rev Psychol. 2018;69: 437-58.

30 Holt-Lunstad J, Smith TB. Loneliness and social isolation as risk factors for CVD: implications for evidence-based patient care and scientific inquiry. Heart. 2016;102(13):987-9.

31 Holt-Lunstad J, Smith TB, Layton JB. Social relationships and mortality risk: a meta-analytic review. PLoS Med. 2010;7(7):e1000316.

32 Duiverman ML, Wempe JB, Bladder G, Vonk JM, Zijlstra JG, Kerstjens HA, et al. Two-year home-based nocturnal noninvasive ventilation added to rehabilitation in chronic obstructive pulmonary disease patients: a randomized controlled trial. Respir Res. 2011;12: 112 .
33 Janssens JP, Breitenstein E, Rochat T, Fitting JW. Does the "oxygen cost diagram" reflect changes in six minute walking distance in follow up studies? Respir Med. 1999;93(11): $810-5$.

34 Morelot-Panzini C, Perez T, Sedkaoui K, de Bock E, Aguilaniu B, Devillier P, et al. The multidimensional nature of dyspnoea in amyotrophic lateral sclerosis patients with chronic respiratory failure: air hunger, anxiety and fear. Respir Med. 2018;145:1-7.

35 Oga T, Taniguchi H, Kita H, Tsuboi T, Tomii $\mathrm{K}$, Ando M, et al. Comparison of different disease-specific health-related quality of life measurements in patients with long-term noninvasive ventilation. Can Respir J. 2017; 2017:8295079.

36 Russo M, Bonanno C, Profazio C, La Foresta $\mathrm{S}$, Faraone $\mathrm{C}$, Lizio A, et al. Which are the factors influencing NIV adaptation and tolerance in ALS patients? Neurol Sci. 2020;42(3): 1023-9.
37 Struik FM, Kerstjens HA, Bladder G, Sprooten R, Zijnen M, Asin J, et al. The Severe Respiratory Insufficiency Questionnaire scored best in the assessment of health-related quality of life in chronic obstructive pulmonary disease. J Clin Epidemiol. 2013;66(10):116674.

38 Pleguezuelos E, Del Carmen A, Moreno E, Ortega P, Vila X, Ovejero L, et al. The experience of COPD patients in lockdown due to the COVID-19 pandemic. Int J Chron Obstruct Pulmon Dis. 2020;15:2621-7.

39 Windisch W, Freidel K, Schucher B, Baumann H, Wiebel M, Matthys H, et al. The Severe Respiratory Insufficiency (SRI) Questionnaire: a specific measure of health-related quality of life in patients receiving home mechanical ventilation. J Clin Epidemiol. 2003; 56(8):752-9. 